\title{
Los medios audiovisuales entre los progenitores y los hijos e hijas
}

\author{
Ferran Casas, Cristina Figuer, Mònica GonzÁlez \\ Y SARA MALO \\ Universitat de Girona
}

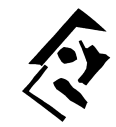

Resumen

En el presente artículo se exponen los resultados de una investigación realizada con una muestra de adolescentes $(N=1211)$ entre 12 y 16 años y otra de sus progenitores $(N=462)$ para explorar cómo las diferentes respuestas generacionales ante la presencia de diversos medios audiovisuales en su entorno próximo afectan las interacciones familiares entre progenitores e bijos/as. Los resultados apuntan al hecho que los progenitores tienden a sobredimensionar tanto el interés como las informaciones de que dispone su propio bijo o bija acerca de la mayor parte de los medios audiovisuales explorados, así como la satisfacción que proporcionan las conversaciones con los adultos acerca de cualquier actividad con estos medios. Los progenitores realizan atribuciones diferentes sobre el uso de medios audiovisuales según se refieran a un bijo o a una bija. Se aprecia una importante diferencia entre la satisfacción con las conversaciones que los y las adolescentes mantienen con sus iguales y la que proporcionan las conversaciones con los adultos respecto a cualquiera de sus actividades con los medios.

Palabras clave: Medios audiovisuales, adolescencia, relaciones intergeneracionales, diferencias de género, comunicación, satisfacción con las conversaciones.

\section{Audiovisual media between parents and their sons and daughters}

\footnotetext{
Abstract

Results from a survey with a sample of 12 to 16 year-old teenagers $(N=1211)$ and their parents $(N=462)$ are presented. Its aim is to explore different generational answers to questions on how the presence of audiovisual media in the home environment affects interactions between parents and their adolescent children. The results indicate that parents tend to overestimate, on the one hand, their child's interest and knowledge about most of the audiovisual media studied, and on the other, the satisfaction provided by conversations with adults on audiovisual activities. Parents' attributions on the use of audiovisual media were found to differ depending on whether they referred to their son or daughter. Reports show important differences between adolescents' satisfaction with conversations they have with their peers involving audiovisual media activities and those they have with adults on this topic.

Keywords: Audio-visual media, adolescence, intergenerational relationships, gender differences, communication, satisfaction with conversations.

Agradecimientos: Investigación desarrollada a partir del proyecto La influencia de las tecnologías de la información y la comunicación en la vida de chicos/as y adolescentes, y estudio de las interacciones y la comunicación que mantienen con los adultos, con número de referencia BSO2002-03180, financiado por el Ministerio de Ciencia y Tecnología y el Plan Nacional de Investigación Científica e Innovación Tecnológica.

Correspondencia con los autores: Universitat de Girona, Institut de Recerca sobre Qualitat de Vida (IRQV), Facultat de Ciències Econòmiques i Empresarials, Campus Montilivi s/n, 17002 Girona. E-mail: ferran.casas@udg.edu. Tel.+34972418980 Fax.+34972418230.

Original recibido: Febrero, 2006; Aceptado: Mayo, 2007.
} 


\section{Introducción}

El uso que los/las adolescentes hacen de las nuevas tecnologías de la información y la comunicación (NTICs) se está convirtiendo en tema central de nuestro futuro social común, en el ámbito internacional. Es necesario incrementar la comprensión de cómo los propios niños, niñas y adolescentes piensan respecto a las nuevas tecnologías, cómo las utilizan, y de cómo las están relacionando con su propio futuro personal, a través de expectativas, deseos y aspiraciones presentes, y cómo estas tecnologías mediatizan sus relaciones interpersonales con los grupos de iguales y con los adultos (Casas, 1995; 1999).

Los estudiosos de la infancia han olvidado demasiado el papel de los medios en la vida de los niños/as y los/las adolescentes. No obstante, actualmente es imposible entender la infancia y la adolescencia sin tener en cuenta tanto la televisión, como las NTICs (Postman, 1983; 1986; Torres, Conde y Ruiz,, 2002). Además, podríamos argumentar que los chicos y chicas de la sociedad actual están viviendo "la infancia/adolescencia de los medios" - ya que su experiencia, y los significados construidos durante estos períodos de desarrollo, están muy determinados y definidos por los medios electrónicos (Buckingham, 2003).

Las tecnologías audiovisuales, los multimedia, y otras NTICs están influyendo en nuestra vida diaria, en nuestros estilos de vida, en nuestras maneras de entender las relaciones interpersonales y también en nuestras maneras de relacionarnos y comunicarnos con las personas. Además, estimulan nuestras capacidades (cognitivas, emocionales, sensitivas, creativas), a través del uso de nuevas posibilidades: interactividad, realidad virtual, etcétera (Casas, 1995; 1998; 1999; Torres et al., 2002).

Según la perspectiva socio-histórica, la formación de la mente parte de unos sistemas de actividad distribuidos socialmente y mediados tanto social como instrumentalmente (Vygotski, 1982-84; citado en del Río, 1999). Si se producen cambios importantes en estos sistemas socioculturales de actividad se producirán cambios correlativos en la construcción de la conciencia y la mente. Una concreción de dicho planteamiento general es lo que podemos observar en los efectos de los medios de comunicación en las nuevas generaciones (del Río, 1999), de manera congruente con las hipótesis formuladas por McLuhan (1977).

Así mismo, del Río, Álvarez y del Río (2004) apuntan cómo desde una perspectiva evolutiva del estudio de los efectos de los medios sobre el desarrollo del ciclo vital de la persona, podemos diferenciar tres grandes tipos: los efectos cognitivos, los efectos directivos y los efectos en los sistemas de actividad y la calidad del contexto ecológico de desarrollo. En cuanto al primero y al segundo -más vinculados a la presente investigación- entendemos, por una parte, que los efectos cognitivos se refieren a todos aquellos cambios producidos por los medios en los sistemas de representación y las capacidades intelectuales y, por otra parte, los efectos directivos son los cambios producidos en la actitud, el juicio moral y la identidad personal, social y cultural, entre otros.

Las nuevas tecnologías y, particularmente las NTICs, nos están ofreciendo nuevas herramientas para mejorar nuestros estilos de vida. Algunos autores han señalado como algunas de estas herramientas pueden permitir que establezcamos nuevas maneras de relacionarnos (Bressand y Distler, 1995). De hecho, estas nuevas formas de relación y comunicación con los otros ya se dan en estos momentos y no sólo en lo que se denomina el ciberespacio. También aparecen en el entorno de los medios audiovisuales: después de utilizarlos, podemos hablar y/o relacionarnos con los otros de nuevas maneras, según nuestra experiencia con cada uno de los medios. 
Algunas investigaciones recientes han explorado cómo los adultos y los chicos/as (particularmente los progenitores y sus hijos/as) se comunican entre ellos cuando se refieren a la televisión y las NTICs. Aún cuando las actividades con los medios audiovisuales son altamente motivadoras para los chicos/as, los resultados de las investigaciones muestran que a menudo no existe verdadera comunicación en estos aspectos, debido a diferentes razones: miedos, informaciones sesgadas, y las sensaciones de incompetencia de los adultos (Llull, 1980). Sucede que la comunicación sobre este tipo de actividades resulta a menudo insatisfactoria para los chicos y chicas (Casas et al., 2000; Casas, Figuer, González y Alsinet, 2002). Por eso, algunos autores (Ohmae, 1995) expresan su preocupación por el creciente desfase generacional en el uso de los medios. Otros como Buckingham (2000) o Papert (1996), en cambio, ven las actividades con estos nuevos medios como una forma de "potenciar" e, incluso, "liberar" a los niños/as. Los defensores de esta última tesis, lejos de apremiar a los adultos para que reafirmen su autoridad sobre los jóvenes, hacen un llamamiento a los adultos para que "escuchen" a sus hijos/as y "se pongan a su nivel".

La investigación aquí presentada partió del interés de estudiar, desde la perspectiva de los chicos y chicas de 12 a 16 años y la de sus progenitores, hoy y en nuestro contexto sociocultural, tanto los equipamientos y facilidades audiovisuales de los que disponen, como el entorno psicosocial de su uso, es decir, con quién hablan y qué grado de satisfacción obtienen con las conversaciones acerca de las actividades con dichos medios. Se trata de una investigación exploratoria y descriptiva, por lo que se planteó que la muestra fuera muy amplia.

Los objetivos específicos son los siguientes:

a) Conocer el grado de información e interés que los adolescentes, por una parte, y sus propios progenitores, por otra, manifiestan tener hacia distintos equipamientos y facilidades audiovisuales, así como el atribuido a los propios hijos e hijas por parte de los progenitores.

b) Conocer los distintos usos que hacen del ordenador y de Internet tanto los adolescentes como sus progenitores.

c) Comparar las evaluaciones que unos y otros hacen de los distintos medios audiovisuales, a partir de diversos criterios de valor.

d) Explorar la frecuencia de la comunicación entre padres e hijos en torno a distintos temas, incluidas actividades con los medios audiovisuales, en función de las percepciones de los adolescentes y de las de los propios progenitores.

e) Explorar la satisfacción proporcionada por las conversaciones en torno a actividades con los medios audiovisuales, mantenidas con distintas personas, tanto la evaluada por chicos y chicas, como la atribuida por los progenitores.

Un objetivo transversal a todos los anteriores ha sido comprobar si existen diferencias de género, tanto en las respuestas de chicos y chicas, como en las atribuciones que los progenitores hacen según lo sean de un hijo o de una hija.

\section{Método}

Para seleccionar a los participantes del estudio se diseñó un muestreo por conglomerados de un conjunto de centros de Educación Secundaria Obligatoria (ESO) de la provincia de Girona, emplazados en la capital o en poblaciones de tamaño intermedio, por considerar que con los datos obtenidos mediante este procedimiento podríamos disponer de una visión general de los comportamientos relacionales de los y las adolescentes entre 12 y 16 años ante los medios audiovisuales. El muestreo por conglomerados tiene la ventaja de que permite obtener una muestra grande de sujetos con características relativamente homogéneas, lo cual abre la posibilidad de explorar los valores de las variables vincula- 
das a cada uno de nuestros objetivos específicos disponiendo de subconjuntos de sujetos suficientes, a fin de poder analizar posteriormente relaciones entre variables.

Por otra parte, un muestreo grande resultaba imprescindible para conseguir una muestra suficiente de progenitores para los análisis con datos apareados, ya que es sobradamente conocida la importante tasa de sujetos que no responden cuando los cuestionarios se remiten por correo. No se persiguió, por tanto, una representatividad de la muestra en relación a la población general, sino un muestreo psicosocial. Presumiblemente la muestra recoge sobre todo sujetos de familias de clase media-baja a media-alta, quedando excluidas las de niveles socioeconómicos situadas en ambos extremos y las de entornos rurales.

De la lista de centros de ESO de la provincia, seleccionamos siete aleatoriamente. Cuatro institutos no aceptaron participar en el estudio y los sustituimos también aleatoriamente.

Informamos a cada uno de los centros que aceptaron cooperar, de nuestros objetivos de investigación a través del director de centro y del presidente/a de la Asociación de Madres y Padres de Alumnos (AMPAs).

La muestra de adolescentes está formada por 1211 estudiantes de los cuatro cursos de la ESO: 557 chicos (46\%) y 654 chicas (54\%). Un 12,6\% tiene 12 años, un $22,3 \%$ tiene 13 , un $26,2 \%$ tiene 14 , un $25,5 \%$ tiene 15 , y un $15,4 \%$ tiene 16 , siendo la media de edad del total de la muestra de $14,07(d t=1,256)$. El menor porcentaje de chicos y chicas que como era de esperar se concentran en las edades de 12 y 16 años, es debido a que cada curso escolar incorpora alumnos de dos edades consecutivas.

La muestra de progenitores está formada por 462 sujetos, de los cuales 179 lo son de chicos (un $38,7 \%$ ) y 238 de chicas $(61,3 \%)$.

\section{Instrumentos}

Se utilizaron dos cuestionarios: uno para adolescentes y otro para sus progenitores.

El cuestionario para adolescentes incluía preguntas dirigidas sistemáticamente a explorar diferentes actividades, opiniones y evaluaciones relacionadas con seis medios audiovisuales (televisor, ordenador, Internet, videoconsola, videojuegos para ordenador y teléfono móvil). También incluía un amplio abanico de posibles temáticas de conversación, entre las que figuraban distintos medios audiovisuales, con el propósito de explorar hasta qué punto las conversaciones entre progenitores e hijo o hija sobre esta temáticas son frecuentes. Finalmente, otra batería de ítems exploró hasta qué punto resultan satisfactorias las conversaciones con distintos interlocutores cuando se refieren a actividades con distintos medios audiovisuales.

El cuestionario para progenitores incluía preguntas equivalentes a las del cuestionario de los/las adolescentes, para poder comparar las respuestas obtenidas. A los progenitores les preguntamos sobre aspectos referidos a su interés actual por los medios audiovisuales, el nivel de información disponible sobre cada uno de los medios, sus atribuciones sobre el nivel de interés e información de su propio hijo/a, la frecuencia con la que conversan con su hijo/a sobre los medios (y también sobre otros temas) y el grado de satisfacción que creían que sus hijo/a tiene respeto a las conversaciones mantenidas sobre estos medios con diferentes personas. Hemos explorado sistemáticamente si sus respuestas han sido distintas según se refirieran a un hijo o a una hija. 


\section{Procedimiento}

Una vez acordada la participación de cada centro de la muestra, seleccionamos aleatoriamente un grupo de cada curso de ESO, de $1^{\circ}$ a $4^{\circ}$, y solicitamos la cooperación del profesor/a responsable de la respectiva aula. Se pidió a los chicos y chicas su cooperación, dejando claro que podían optar libremente por no participar y se les informó de que los datos serían tratados de manera confidencial.

Los cuestionarios se administraron de manera grupal en su aula habitual. Uno de sus profesores/as y una o dos de las investigadoras estuvieron presentes durante la administración de los cuestionarios para resolver cualquier duda. Cada sesión duró alrededor de una hora para los chicos y chicas más jóvenes y entre 35 40 minutos para los mayores.

Al final de la sesión dimos a cada uno de los alumnos una carta y un cuestionario para sus progenitores en un sobre, para que lo entregaran en mano. Les pedimos que lo devolvieran a su profesor en el plazo de una semana, en sobre cerrado.

El cuestionario de progenitores tenía un código, para poderlo aparear con el de su hijo/a. Por nuestra experiencia pasada, sabíamos que los progenitores a veces responden de manera individual y a veces de forma conjunta, por lo que el cuestionario para progenitores incluyó un pregunta sobre quién respondía, con tres opciones de respuesta: el padre, la madre o ambos conjuntamente. El número de padres varones que han respondido solos es muy bajo, por lo que no hemos considerado oportuno profundizar en el análisis de las respuestas de los progenitores controlando el sexo de los mismos.

\section{Resultados}

Hemos considerado que las diferencias de edad no merecen ser destacadas entre los resultados de este estudio debido a que para el conjunto de temáticas exploradas entre los 12 y los 16 años se han observado muy pocas diferencias significativas que en ningún caso tenían una tendencia clara. Por este motivo, los resultados se exponen teniendo en cuenta el género como eje del análisis.

\section{Nivel de interés e información por los medios audiovisuales}

La tabla I muestra las medias de las puntuaciones que chicos/as han dado a su interés por los medios, el propio interés de sus progenitores teniendo en cuenta si lo son de un chico o de una chica, así como el interés que padres y madres atribuyen a su hijo o hija. Las respuestas se han obtenido mediante una escala tipo Likert del 1 (Nada) al 5 (Mucho).

Los chicos manifiestan estar significativamente más interesados por el ordenador $\left(t_{1120}=2,491 ; p=0,013\right)$, la videoconsola $\left(t_{1129}=20,803 ; p<0,0005\right)$ y los videojuegos para ordenador $\left(t_{1074}=14,789 ; p<0,0005\right)$ que las chicas, mientras que estas últimas muestran un mayor interés por el teléfono móvil $\left(t_{1053}=8,052\right.$; $p<0,0005)$.

Al comparar las respuestas de progenitores y adolescentes, lo que más llama la atención es que los primeros informan de un interés claramente inferior por absolutamente todos los medios audiovisuales que su propio hijo o hija, siendo estas diferencias significativas para todos los medios estudiados: televisión $\left(t_{446}=\right.$ $14,183 ; p<0,0005)$, ordenador $\left(t_{462}=10,446 ; p<0,0005\right)$, Internet $\left(t_{446}=\right.$ $14,897 ; p<0,0005)$, videoconsola $\left(t_{447}=14,892 ; p<0,0005\right)$, videojuegos para ordenador $\left(t_{456}=15,462 ; p<0,0005\right)$ y móvil $\left(t_{462}=13,122 ; p<0,0005\right)$. 
TABLA I

Nivel de interés de los/las adolescentes y sus progenitores y atribuciones de los progenitores respecto al de sus bijos/as

\begin{tabular}{|c|c|c|c|c|c|c|c|}
\hline MEDIAS & & Televisión & Ordenador & Internet & $\begin{array}{l}\text { Vídeo- } \\
\text { consola }\end{array}$ & $\begin{array}{c}\text { Video- } \\
\text { juegos } \\
\text { para } \\
\text { ordenador }\end{array}$ & Móvil \\
\hline $\begin{array}{l}\text { Interés de los } \\
\text { chicos/as }\end{array}$ & $\begin{array}{l}\text { Cbico } \\
\text { Chica } \\
\text { Total }\end{array}$ & $\begin{array}{l}3,49 \\
3,50 \\
3,49\end{array}$ & $\begin{array}{l}3,69 \\
3,53 \\
3,60\end{array}$ & $\begin{array}{l}3,64 \\
3,74 \\
3,70\end{array}$ & $\begin{array}{l}3,31 \\
1,81 \\
2,50\end{array}$ & $\begin{array}{l}3,14 \\
2,05 \\
2,55\end{array}$ & $\begin{array}{l}3,34 \\
3,95 \\
3,67\end{array}$ \\
\hline $\begin{array}{l}\text { Interés de los } \\
\text { progenitores }\end{array}$ & $\begin{array}{l}\text { Chico } \\
\text { Chica } \\
\text { Total }\end{array}$ & $\begin{array}{l}2,71 \downarrow \\
2,88 \downarrow \\
2,81 \downarrow\end{array}$ & $\begin{array}{l}2,88 \downarrow \\
3,00 \downarrow \\
2,95 \downarrow\end{array}$ & $\begin{array}{l}2,62 \downarrow \\
2,73 \downarrow \\
2,69 \downarrow\end{array}$ & $\begin{array}{l}1,32 \downarrow \\
1,35 \downarrow \\
1,34 \downarrow\end{array}$ & $\begin{array}{l}1,38 \downarrow \\
1,48 \downarrow \\
1,44 \downarrow\end{array}$ & $\begin{array}{l}2,55 \downarrow \\
2,74 \downarrow \\
2,66 \downarrow\end{array}$ \\
\hline $\begin{array}{l}\text { Atribución } \\
\text { que hacen los } \\
\text { progenitores } \\
\text { del interés de } \\
\text { su hijo o hija }\end{array}$ & $\begin{array}{l}\text { Cbico } \\
\text { Cbica }\end{array}$ & $\begin{array}{l}3,18 \downarrow \\
3,33 \downarrow\end{array}$ & $\begin{array}{l}3,67 \downarrow \\
3,78 \uparrow \\
3,74 \uparrow\end{array}$ & $\begin{array}{l}3,53 \downarrow \\
3,72 \downarrow \\
3,64 \downarrow\end{array}$ & $\begin{array}{l}3,40 \uparrow \\
1,96 \uparrow \\
2,52 \uparrow\end{array}$ & $\begin{array}{l}3,23 \uparrow \\
2,27 \uparrow \\
2,64 \uparrow\end{array}$ & $\begin{array}{l}3,22 \downarrow \\
3,79 \downarrow \\
3,57 \downarrow\end{array}$ \\
\hline
\end{tabular}

- Las flechas $\downarrow$ indican que las puntuaciones medias obtenidas por los progenitores son menores que las obtenidas por sus hijos/as sin indicar significación estadística.

- Las flechas $\uparrow$ indican que puntuaciones medias obtenidas por los progenitores son mayores que las obtenidas por sus hijos/as sin indicar significación estadística.

Curiosamente, los progenitores de chicas muestran mayor interés por todos los medios que los de los chicos aunque sólo en el caso de la televisión las diferencias alcanzan la significación $\left(t_{467}=2,154 ; p=0,032\right)$.

Los progenitores consideran que su interés por todos los medios estudiados es significativamente más bajo que el interés que tienen sus hijos/as por estos mismos medios (televisión: $t_{461}=10,443 ; p<0,0005$, ordenador: $t_{459}=12,672 ; p<$ 0,0005 , Internet: $t_{439}=14,023 ; p<0,0005$, videoconsola: $t_{441}=19,033 ; p<$ 0,0005 , videojuegos para ordenador: $t_{446}=20,276 ; p<0,0005$ y móvil: $t_{461}=$ $13,678 ; p<0,0005)$.

Se observa que los progenitores de los chicos atribuyen a sus hijos mayor interés acerca de la videoconsola y los videojuegos para ordenador $\left(t_{449}=13,344 ; p<\right.$ 0,0005 y $t_{451}=8,500 p<0,0005$, respectivamente), que los progenitores de las chicas. En cambio, estos últimos atribuyen a sus hijas mayor interés por el teléfono móvil de lo que los padres y madres de los chicos atribuyen a sus hijos $\left(t_{463}=\right.$ $5,191 ; p<0,0005)$.

En la tabla II se muestran las puntuaciones medias de adolescentes y de progenitores sobre la información que manifiestan disponer acerca cada uno de los medios estudiados (diferenciando para estos últimos si se trata de los progenitores de una chica o de un chico), así como la información que los progenitores atribuyen a su hijo o hija. Las respuestas se han recogido mediante una escala tipo Likert del 1 (Nada) al 5 (Mucha).

Los adolescentes varones manifiestan estar significativamente más informados respecto a los videojuegos $\left(t_{984}=16,158 ; p<0,0005\right)$ y los juegos de ordena$\operatorname{dor}\left(t_{1034}=11,556 ; p<0,0005\right)$ y las chicas respecto al teléfono móvil $\left(t_{1110}=\right.$ $5,752 ; p<0,0005)$.

Los progenitores, independientemente de si lo son de un chico o una chica, manifiestan estar significativamente menos informados que sus hijos/as en relación a los siguientes medios: ordenador $\left(t_{461}=5,701 ; p<0,0005\right)$, Internet $\left(t_{448}=\right.$ $12,107 ; p<0,0005)$ y móvil $\left(t_{457}=5,030 ; p<0,0005\right)$. 
TABLA II

Nivel de información de los/las adolescentes y sus progenitores y atribuciones de sus progenitores respecto al de sus hijos/as

\begin{tabular}{|c|c|c|c|c|c|c|c|}
\hline MEDIAS & & Televisión & Ordenador & Internet & $\begin{array}{l}\text { Vídeo- } \\
\text { consola }\end{array}$ & $\begin{array}{c}\text { Video } \\
\text { juegos } \\
\text { para } \\
\text { ordenador }\end{array}$ & Móvil \\
\hline $\begin{array}{l}\text { Información de } \\
\text { los chicos/as }\end{array}$ & $\begin{array}{l}\text { Chico } \\
\text { Chica } \\
\text { Total }\end{array}$ & $\begin{array}{l}3,37 \\
3,30 \\
3,33\end{array}$ & $\begin{array}{l}3,30 \\
3,19 \\
3,24\end{array}$ & $\begin{array}{l}3,57 \\
3,53 \\
3,55\end{array}$ & $\begin{array}{l}2,86 \\
1,68 \\
2,23\end{array}$ & $\begin{array}{l}2,78 \\
1,92 \\
2,32\end{array}$ & $\begin{array}{l}3,16 \\
3,62 \\
3,41\end{array}$ \\
\hline $\begin{array}{l}\text { Información de } \\
\text { los progenitores }\end{array}$ & $\begin{array}{l}\text { Chico } \\
\text { Chica } \\
\text { Total }\end{array}$ & $\begin{array}{l}3,38 \uparrow \\
3,40 \uparrow \\
3,39 \uparrow\end{array}$ & $\begin{array}{l}2,94 \downarrow \\
2,80 \downarrow \\
2,85 \downarrow\end{array}$ & $\begin{array}{l}2,64 \downarrow \\
2,60 \downarrow \\
2,62 \downarrow\end{array}$ & $\begin{array}{l}2,38 \downarrow \\
2,07 \uparrow \\
2,19 \downarrow\end{array}$ & $\begin{array}{l}2,33 \downarrow \\
2,20 \uparrow \\
2,25 \downarrow\end{array}$ & $\begin{array}{l}2,87 \downarrow \\
2,97 \downarrow \\
2,93 \downarrow\end{array}$ \\
\hline $\begin{array}{l}\text { Atribución que } \\
\text { hacen los } \\
\text { progenitores } \\
\text { del interés de } \\
\text { su hijo o hija }\end{array}$ & $\begin{array}{l}\text { Chico } \\
\text { Chica }\end{array}$ & $\begin{array}{l}3,44 \uparrow \\
3,54 \uparrow\end{array}$ & $\begin{array}{l}3,49 \uparrow \\
3,56 \uparrow\end{array}$ & $\begin{array}{l}3,28 \downarrow \\
3,36 \downarrow\end{array}$ & $\begin{array}{l}3,67 \uparrow \\
2,73 \uparrow\end{array}$ & $\begin{array}{l}3,53 \uparrow \\
2,86 \uparrow\end{array}$ & $\begin{array}{l}3,30 \uparrow \\
3,71 \uparrow\end{array}$ \\
\hline
\end{tabular}

La única diferencia estadísticamente significativa entre la información de la que disponen los progenitores, según lo sean de un chico o de una chica, se da en las videoconsolas $\left(t_{456}=2,773 ; p=0,006\right)$, a favor de los primeros.

Los progenitores atribuyen a su hijo o hija mayor información de la que el propio progenitor manifiesta disponer acerca de todos los medios audiovisuales estudiados (televisión: $t_{470}=2,650 ; p<0,0005$, ordenador: $t_{460}=13,253 ; p<$ 0,0005 , Internet: $t_{457}=12,671 ; p<0,0005$, videoconsola: $t_{450}=14,236 ; p<$ 0,0005 , videojuegos para ordenador: $t_{448}=14,107 ; p<0,0005$ y móvil: $t_{457}=$ $10,373 ; p<0,0005)$.

Si tenemos en cuenta la atribución que hacen los progenitores en función del género del hijo/a, observamos que los progenitores de los chicos atribuyen mayor información a su hijo de lo que los progenitores de chicas atribuyen a su hija acerca de la videoconsola $\left(t_{455}=8,473 ; p<0,0005\right)$ y los videojuegos para ordenador $\left(t_{457}=6,228 p<0,0005\right)$. Lo contrario pasa en relación al móvil $\left(t_{461}=\right.$ $3,732 ; p<0,0005)$.

\section{Diferentes usos del ordenador e Internet}

En las tablas III y IV se muestran las puntuaciones medias que chicos/as y sus progenitores han dado a la frecuencia de uso de diferentes utilidades del ordenador e Internet. En ambos casos las respuestas se han obtenido mediante escalas tipo Likert del 1 (Nunca) al 5 (Muy a menudo).

Han manifestado utilizar el ordenador un 93,6\% de los y las adolescentes y el $64,2 \%$ de sus progenitores. Las actividades que los chicos varones informan que realizan significativamente con más frecuencia que las chicas son las de editar o componer música $\left(t_{978}=4,554 ; p<0,0005\right)$, la de jugar con juegos $\left(t_{1114}=\right.$ $12,977 ; p<0,0005)$ y la de mirar películas en DVD con el ordenador $\left(t_{994}=\right.$ $4,103 ; p<0,0005)$, mientras que las chicas dicen utilizar más a menudo que los chicos las funciones de escribir $\left(t_{1010}=10,523 ; p<0,0005\right)$ y la de escuchar músi$\mathrm{ca}\left(t_{1050}=4,918 ; p<0,0005\right)$.

Observamos que los progenitores utilizan todas las funciones exploradas con menor frecuencia que su hijo o hija (escribir: $t_{288}=5,403 ; p<0,0005$, dibujar, pintar, diseñar: $t_{265}=3,175 ; p=0,002$, escuchar música: $t_{267}=17,555 ; p<$ 
TABLA III

Usos del ordenador por parte de los/las adolescentes y sus progenitores

\begin{tabular}{|c|c|c|c|c|c|c|c|}
\hline MEDIAS & & $\begin{array}{l}\text { Escribir } \\
\text { pintar } \\
\text { diseñar }\end{array}$ & Dibujar & $\begin{array}{l}\text { Escuchar } \\
\text { música }\end{array}$ & $\begin{array}{l}\text { Editaro } \\
\text { componer } \\
\text { música }\end{array}$ & $\begin{array}{c}\text { Jugar con } \\
\text { juegos }\end{array}$ & $\begin{array}{c}\text { Mirar } \\
\text { películas } \\
\text { en DVD }\end{array}$ \\
\hline \multirow{3}{*}{$\begin{array}{l}\text { Uso que hacen } \\
\text { los/las } \\
\text { adolescentes } \\
\text { del ordenador }\end{array}$} & Chico & 3,30 & 2,08 & 3,42 & 2,08 & 3,61 & 2,37 \\
\hline & Chica & 3,97 & 2,15 & 3,82 & 1,72 & 2,61 & 2,02 \\
\hline & Total & 3,66 & 2,12 & 3,64 & 1,88 & 3,06 & 2,18 \\
\hline \multirow{3}{*}{$\begin{array}{l}\text { Uso que hacen } \\
\text { los progenitores } \\
\text { del ordenador }\end{array}$} & Chico & $3,58 \uparrow$ & $1,89 \downarrow$ & $2,03 \downarrow$ & $1,25 \downarrow$ & $1,75 \downarrow$ & $1,44 \downarrow$ \\
\hline & Chica & $3,53 \downarrow$ & $1,97 \downarrow$ & $2,04 \downarrow$ & $1,25 \downarrow$ & $1,80 \downarrow$ & $1,40 \downarrow$ \\
\hline & Total & $3,55 \downarrow$ & $1,94 \downarrow$ & $2,04 \downarrow$ & $1,25 \downarrow$ & $1,78 \downarrow$ & $1,41 \downarrow$ \\
\hline
\end{tabular}

0,0005, editar o componer música: $t_{261}=7,636 ; p<0,0005$, jugar con juegos: $t_{274}=12,957 ; p<0,0005$ y mirar películas en DVD: $\left.t_{268}=7,561 ; p<0,0005\right)$. No existen diferencias significativas entre los progenitores de un chico y los de una chica.

TABLA IV

Usos de Internet por parte de los/las adolescentes y sus progenitores

\begin{tabular}{lccccccc}
\hline MEDIAS & & $\begin{array}{c}\text { Buscar } \\
\text { información }\end{array}$ & Navegar & $\begin{array}{c}\text { Correo } \\
\text { electrónico }\end{array}$ & $\begin{array}{c}\text { Chatear } \\
\text { (bablar) }\end{array}$ & $\begin{array}{c}\text { Descargar } \\
\text { música }\end{array}$ & $\begin{array}{c}\text { Jugara } \\
\text { juegos } \\
\text { on-line }\end{array}$ \\
\hline $\begin{array}{l}\text { Usos que } \\
\text { hacen los/las } \\
\text { adolescentes } \\
\text { de Internet }\end{array}$ & Chico & 3,28 & 3,43 & 2,88 & 3,36 & 3,41 & 2,67 \\
\hline $\begin{array}{l}\text { Uso que } \\
\text { hacen los } \\
\text { progenitores } \\
\text { de Internet }\end{array}$ & Chica & 3,43 & 3,38 & 3,34 & 3,79 & 3,02 & 1,88 \\
\hline & Chica & $3,58 \uparrow$ & 3,40 & 3,13 & 3,60 & 3,20 & 2,24 \\
\hline
\end{tabular}

\section{Internet}

Un $86,1 \%$ de los chicos y chicas y el $53 \%$ de sus progenitores han manifestado utilizar Internet.

Los chicos varones utilizan significativamente con mayor frecuencia las funciones de descargar música o juegos $\left(t_{995}=4,060 ; p<0,0005\right)$ y jugar a juegos on-line $\left(t_{852}=9,368 ; p<0,0005\right)$, mientras que las chicas lo hacen con el chatear $\left(t_{967}=4,576 ; p<0,0005\right)$, mandar correos electrónicos $\left(t_{1030}=4,810 ; p<0,0005\right)$ y buscar información $\left(t_{1043}=2,210 ; p=0,027\right)$.

Los progenitores manifiestan utilizar con menor frecuencia que su propio hijo o hija las funciones de Internet exploradas. Todas ellas, exceptuando la de buscar información, muestran diferencias significativas (navegar: $t_{218}=10,417 ; p<$ 0,0005, correo electrónico: $t_{220}=3,338 ; p=0,001$, chatear: $t_{219}=19,818 ; p<$ 0,0005 , descargar música: $t_{219}=14,129 ; p<0,0005$ y jugar a juegos on-line: $t_{221}$ $=8,267 ; p<0,0005)$.

No hay diferencias significativas en los usos que los padres hacen de Internet según si son progenitores de un chico o de una chica. 
Evaluaciones de las experiencias con diferentes medios audiovisuales

A continuación se presentan las puntuaciones medias de las evaluaciones que chicos y chicas han realizado de distintas experiencias con cada medio explorado y las atribuciones que hacen los progenitores respecto a las mismas experiencias de su propio hijo o hija (Tablas V, VI, VII, VIII, IX, X), mediante escalas bipolares de 5 puntos.

TABLA V

Evaluación del aburrimiento y/o la diversión con el uso de medios de los adolescentes y atribución que los progenitores hacen a sus bijos/as

Sensación de aburrimiento / Diversión

\begin{tabular}{llcccc}
\hline MEDIAS & & Televisión & Ordenador & Internet & Videojuegos \\
\hline Adolescentes & Chico & 3,77 & 3,98 & 4,04 & 4,23 \\
& Chica & 3,82 & 3,70 & 4,09 & 2,72 \\
& Total & 3,80 & 3,83 & 4,06 & 3,42 \\
\hline \multirow{2}{*}{ Atribución } & Chico & $3,61 \downarrow$ & $4,03 \uparrow$ & $3,92 \downarrow$ & $4,19 \downarrow$ \\
de los progenitores & Chica & $3,80 \downarrow$ & $3,96 \uparrow$ & $3,85 \downarrow$ & $3,07 \uparrow$ \\
& Total & $3,70 \downarrow$ & $3,99 \uparrow$ & $3,88 \downarrow$ & $4,41 \uparrow$ \\
\hline
\end{tabular}

En primer lugar se pidió que evaluaran el aburrimiento frente a la diversión que los/las adolescentes experimentan con cada medio (Tabla V). Los chicos han manifestado que se divierten significativamente más que las chicas con el ordenador $\left(t_{1187}=4,063 ; p<0,0005\right)$ y los videojuegos $\left(t_{1149}=19,988 ; p<\right.$ $0,0005)$.

Los progenitores, por su parte, piensan que su hijo o hija se divierte ante Internet menos de lo que el o la adolescente informa $\left(t_{423}=2,436 ; p=\right.$ $0,015)$. En cambio, tienen la percepción de que su hijo/a se divierte ante el ordenador y los videojuegos más de lo que en realidad sucede $\left(t_{461}=4,466 ; p\right.$ $<0,0005$ y $t_{436}=2,674 ; p=0,008$, respectivamente).

En relación al género del hijo o hija, las diferencias en las respuestas de los progenitores son estadísticamente significativas únicamente por lo que respecta a los videojuegos $\left(t_{440}=9,116 ; p<0,0005\right)$. Los progenitores de los chicos atribuyen mayor diversión con los videojuegos a sus hijos que los progenitores de las chicas a sus hijas.

TABLA VI

Evaluación del no aprendizaje y/o aprendizaje con el uso de medios de los adolescentes y atribución que los progenitores bacen a sus bijos/as

\begin{tabular}{lccccc}
\hline & \multicolumn{5}{c}{ Noaprendizaje / Aprendizaje } \\
\hline MEDIAS & & Televisión & Ordenador & Internet & Videojuegos \\
\hline Adolescentes & Chico & 3,22 & 3,50 & 3,69 & 2,37 \\
& Chica & 3,09 & 3,32 & 3,49 & 1,53 \\
& Total & 3,15 & 3,40 & 3,58 & 1,92 \\
\hline Atribución & Chico & $2,64 \downarrow$ & $3,79 \uparrow$ & $3,54 \downarrow$ & $2,12 \downarrow$ \\
de los & Chica & $2,69 \downarrow$ & $3,92 \uparrow$ & $3,62 \uparrow$ & $1,90 \uparrow$ \\
progenitores & Total & $2,67 \downarrow$ & $3,87 \uparrow$ & $3,59 \uparrow$ & $1,99 \uparrow$ \\
\hline
\end{tabular}


En relación al menor o mayor aprendizaje que los/las adolescentes pueden experimentar con el uso de los diferentes medios (Tabla VI), se observa como los chicos manifiestan significativamente que aprenden más que las chicas con los cuatro medios por los que preguntamos: la televisión $\left(t_{1141}=1,972 ; p=0,049\right)$, el ordenador $\left(t_{1193}=2,473 ; p=0,014\right)$, Internet $\left(t_{1175}=2,816 ; p=0,005\right)$ y los videojue$\operatorname{gos}\left(t_{972}=12,381 ; p<0,0005\right)$.

Los adolescentes manifiestan aprender con la televisión significativamente más de lo que sus progenitores piensan $\left(t_{463}=7,030 ; p<0,0005\right)$, mientras que con el ordenador ocurre todo lo contrario $\left(t_{457}=8,509 ; p<0,0005\right)$. No hay diferencias significativas entre las respuestas de los progenitores de los chicos y los de las chicas sobre la atribución que hacen del aprendizaje obtenido por los adolescentes.

TABLA VII

Evaluación de la pérdida y/o aprovechamiento del tiempo con el uso de medios de los adolescentes y atribución que los progenitores hacen a sus bijos/as

Pérdida de tiempo / Aprovechamiento de tiempo

\begin{tabular}{lccccc}
\hline MEDIAS & & Televisión & Ordenador & Internet & Videojuegos \\
\hline Adolescentes & Chico & 2,78 & 3,45 & 3,44 & 2,74 \\
& Chica & 2,69 & 3,46 & 3,38 & 1,68 \\
& Total & 2,73 & 3,45 & 3,41 & 2,17 \\
\hline Atribución & Chico & $2,37 \downarrow$ & $3,68 \uparrow$ & $3,36 \downarrow$ & $2,01 \downarrow$ \\
de los & Chica & $2,44 \downarrow$ & $3,85 \uparrow$ & $3,48 \uparrow$ & $1,75 \uparrow$ \\
progenitores & Total & $2,41 \downarrow$ & $3,79 \uparrow$ & $3,43 \uparrow$ & $1,85 \downarrow$ \\
\hline
\end{tabular}

En cuanto a la sensación de pérdida o aprovechamiento del tiempo que los chicos y las chicas experimentan con estos medios audiovisuales (Tabla VII), hemos observado como los chicos perciben que aprovechan de manera significativa más el tiempo que las chicas con los videojuegos $\left(t_{1124}=13,847 ; p<0,0005\right)$.

Para los progenitores, su hijo o hija pierde el tiempo con la televisión $\left(t_{461}=\right.$ $4,259 ; p<0,0005)$ y los videojuegos $\left(t_{436}=3,105 ; p=0,002\right)$, significativamente menos de lo que él o ella considera, mientras que con el ordenador ocurre todo lo contrario $\left(t_{459}=5,038 ; p<0,0005\right)$.

La atribución de aprovechamiento del tiempo en el uso de los videojuegos es significativamente más elevada entre los progenitores de los chicos que entre los progenitores de las chicas $\left(t_{440}=2,624 ; p=0,009\right)$.

TABLA VIII

Evaluación de la no utilidad y/o la utilidad con el uso de medios de los adolescentes y atribución que los progenitores bacen a sus hijos/as

No utilidad / Utilidad

\begin{tabular}{lccccc}
\hline MEDIAS & & Televisión & Ordenador & Internet & Videojuegos \\
\hline Adolescentes & Chico & 3,45 & 4,04 & 4,03 & 2,89 \\
& Chica & 3,31 & 4,14 & 4,11 & 1,75 \\
& Total & 3,38 & 4,09 & 4,07 & 2,28 \\
\hline Atribución & Chico & $2,63 \downarrow$ & $4,09 \uparrow$ & $3,84 \downarrow$ & $1,94 \downarrow$ \\
de los & Chica & $2,70 \downarrow$ & $4,24 \uparrow$ & $3,93 \downarrow$ & $1,80 \uparrow$ \\
progenitores & Total & $2,67 \downarrow$ & $4,18 \uparrow$ & $3,90 \downarrow$ & $1,85 \downarrow$ \\
\hline
\end{tabular}


En relación a la inutilidad o utilidad que los chicos y chicas manifiestan en relación al uso de cada medio (Tabla VIII), podemos apreciar como los chicos informan que experimentan mayor utilidad con la televisión $\left(t_{1193}=1,995 ; p=\right.$ $0,046)$ y con los videojuegos $\left(t_{1015}=15,249 ; p<0,0005\right)$ que las chicas, siendo estas diferencias significativas.

La utilidad del uso de la televisión, de Internet y de los videojuegos para el chico o la chica es considerada significativamente mayor por los adolescentes que por los progenitores (televisión: $t_{459}=9,513 ; p<0,0005$, Internet: $t_{416}=2,998 ; p$ $=0,003$ y videojuegos: $\left.t_{433}=4,816 ; p<0,0005\right)$. No existen diferencias significativas entre las respuestas de los progenitores de chicos y los de chicas.

TABLA IX

Evaluación de la pasividad y/o actividad con el uso de medios de los adolescentes y atribución que los progenitores hacen a sus bijos/as

\begin{tabular}{lccccc}
\hline & \multicolumn{5}{c}{ Pasividad/Actividad } \\
\hline MEDIAS & & Televisión & Ordenador & Internet & Videojuegos \\
\hline Adolescentes & Chico & 2,92 & 3,46 & 3,44 & 3,58 \\
& Chica & 2,89 & 3,30 & 3,49 & 2,36 \\
& Total & 2,90 & 3,38 & 3,47 & 2,93 \\
\hline Atribución & Chico & $2,54 \downarrow$ & $3,73 \uparrow$ & $3,69 \uparrow$ & $3,57 \downarrow$ \\
de los & Chica & $2,69 \downarrow$ & $3,92 \uparrow$ & $3,82 \uparrow$ & $2,68 \uparrow$ \\
progenitores & Total & $2,63 \downarrow$ & $3,85 \uparrow$ & $3,77 \uparrow$ & $3,03 \uparrow$ \\
\hline
\end{tabular}

Con respecto a la sensación de actividad o pasividad de los/las adolescentes frente a estos medios (Tabla IX), observamos como los chicos experimentan significativamente más sensación de actividad que las chicas con el ordenador $\left(t_{1108}=\right.$ $2,035 ; p=0,042)$ y los videojuegos $\left(t_{1174}=14,477 ; p<0,0005\right)$.

Los adolescentes manifiestan sentirse significativamente más pasivos cuando utilizan el ordenador $\left(t_{446}=8,178 ; p<0,0005\right)$ e Internet $\left(t_{413}=4,797 ; p<\right.$ $0,0005)$ de lo que sus progenitores les atribuyen. Los padres y madres de las chicas atribuyen a su hija una sensación de actividad ante los videojuegos significativamente menor que la que los progenitores de los chicos atribuyen a su hijo $\left(t_{390}=6,421 ; p<0,0005\right)$.

TABLA X

Evaluación de la soledad y/o acompañamiento con el uso de medios de los adolescentes y atribución que los progenitores bacen a sus bijos/as

\begin{tabular}{lccccc}
\hline & & \multicolumn{4}{c}{ Soledad/Acompañamiento } \\
\hline MEDIAS & & Televisión & Ordenador & Internet & Videojuegos \\
\hline Adolescentes & Chico & 3,43 & 3,23 & 3,62 & 3,31 \\
& Chica & 3,55 & 3,07 & 3,77 & 2,24 \\
& Total & 3,50 & 3,14 & 3,70 & 2,74 \\
\hline Atribución & Chico & $3,27 \downarrow$ & $3,27 \uparrow$ & $3,37 \downarrow$ & $3,08 \downarrow$ \\
de los & Chica & $3,42 \downarrow$ & $3,33 \uparrow$ & $3,59 \downarrow$ & $2,45 \uparrow$ \\
progenitores & Total & $3,36 \downarrow$ & $3,31 \uparrow$ & $3,51 \downarrow$ & $2,70 \downarrow$ \\
\hline
\end{tabular}

Finalmente, respecto a las evaluaciones que hacen los chicos y las chicas sobre la sensación de soledad o acompañamiento con cada medio (Tabla X), hemos observa- 
do que los chicos manifiestan sentirse significativamente más acompañados con los videojuegos $\left(t_{1178}=13,383 ; p<0,0005\right)$ que las chicas.

Ante el ordenador, chicos y chicas manifiestan sentirse solos en mayor medida de lo que piensan sus progenitores $\left(t_{444}=3,356 ; p=0,001\right)$, mientras que perciben más acompañamiento que el que les atribuyen ante la televisión $\left(t_{449}=\right.$ $2,380 ; p=0,018)$ e Internet $\left(t_{409}=2,636 ; p=0,009\right)$.

Los progenitores de los chicos atribuyen a su hijo una sensación de acompañamiento significativamente mayor que la atribuida a las chicas por parte de sus progenitores $\left(t_{383}=4,877 ; p<0,0005\right)$.

\section{Frecuencia en la comunicación entre padres e hijos}

A continuación se muestran las medias de las puntuaciones dadas para la frecuencia de las conversaciones con cada progenitor acerca de distintos temas, entre los que se han incluido algunas actividades con medios audiovisuales, por parte de los chicos y chicas (Tablas XI y XII). Las respuestas se han medido a través de escalas tipo Likert del 1 (No bablamos nada) al 5 (Son temas muy babituales).

Una simple ojeada a ambas tablas hace patente que los progenitores tienden a considerar más frecuentes que su hijo o hija las conversaciones mantenidas por la madre (Tabla XII) con él o ella sobre cualquier tema, excepto la limpieza y el orden, que son consideradas más frecuentes por los adolescentes. Las diferencias son significativas para todos los temas: (escuela y aprendizajes: $t_{456}=3,954 ; p<$ 0,0005, televisión: $t_{449}=3,773 ; p<0,0005$, limpieza y orden: $t_{442}=2,630 ; p=$ 0,009 , tiempo libre y actividades de ocio: $t_{448}=11,480, p<0,0005$, sentimientos: $t_{451}=9,932 ; p<0,0005$, futuro: $t_{443}=2,703 ; p=0,007$, ordenadores: $t_{446}=$ 9,922; $p<0,0005$, lo que pasa en el mundo: $t_{450}=5,549 ; p<0,0005$, ropa: $t_{443}=$ 6,894; $p<0,0005$, comida: $t_{447}=7,133 ; p<0,0005$, la ciudad o pueblo donde vives: $t_{448}=7,738 ; p<0,0005$, la vida cotidiana: $t_{442}=7,361 ; p<0,0005$, familia y familiares: $t_{448}=6,039 ; p<0,0005$, otras personas y relaciones: $t_{441}=8,276 ; p$ $<0,0005$, videoconsolas y juegos de ordenador: $t_{439}=6,745 ; p<0,0005$, responsabilidad y otros valores morales: $t_{449}=4,534 ; p<0,0005$, religión: $t_{439}=$ 12,246; $p<0,0005$, deportes: $t_{447}=5,670 ; p<0,0005$, música: $t_{445}=6,915 ; p<$ 0,0005 y revistas: $\left.t_{444}=11,269 ; p<0,0005\right)$.

También son significativas para todos los temas las diferencias en la frecuencia informada por los chicos/as y la informada por sus progenitores de las conversaciones mantenidas con el padre (Tabla XI) a excepción de las relativas a la escuela y los aprendizajes, la televisión, el futuro, la ropa y los deportes. En este caso los progenitores consideran menos frecuentes que sus hijos las relacionadas con la limpieza y orden $\left(t_{414}=3,283 ; p=0,001\right)$, y más frecuentes las conversaciones mantenidas sobre: tiempo libre y actividades de ocio $\left(t_{420}=7,590 ; p<0,0005\right)$, sentimientos $\left(t_{416}=5,525 ; p<0,0005\right)$, ordenadores $\left(t_{422}=7,004 ; p<0,0005\right)$, lo que pasa en el mundo $\left(t_{424}=3,759 ; p<0,0005\right)$, comida $\left(t_{416}=2,287 ; p=\right.$ $0,023)$, la ciudad o pueblo donde vives $\left(t_{416}=6,063 ; p<0,0005\right)$, la vida cotidiana $\left(t_{417}=5,209 ; p<0,0005\right)$, familia y familiares $\left(t_{425}=3,075 ; p=0,002\right)$, otras personas y relaciones $\left(t_{416}=7,050 ; p<0,0005\right)$, videoconsolas y juegos de ordenador $\left(t_{419}=4,599 ; p<0,0005\right)$, responsabilidad y otros valores morales $\left(t_{427}=\right.$ $3,244 ; p=0,001)$, religión $\left(t_{420}=10,042 ; p<0,0005\right)$, música $\left(t_{416}=4,966 ; p<\right.$ $0,0005)$ y revistas $\left(t_{422}=7,890 ; p<0,0005\right)$.

Cuando se trata de hablar con el padre sobre diferentes aspectos de la vida de los/las adolescentes (Tabla XI), se observan diferencias estadísticamente significativas en función de si se trata de un chico o de una chica. Las chicas manifiestan hablar más frecuentemente que los chicos con su padre sobre la escuela y los aprendizajes $\left(t_{1182}=4,034 ; p<0,0005\right)$, la limpieza y el orden $\left(t_{1168}=2,461 ; p=\right.$ 
Los medios audiovisuales entre los progenitores y los bijos e hijas / F. Casas et al.

TABLA XI

Frecuencia en la comunicación con el padre sobre diferentes temas según los adolescentes y según sus progenitores

\begin{tabular}{|c|c|c|c|c|c|c|}
\hline \multirow{3}{*}{ MEDIAS } & \multicolumn{6}{|c|}{ Con el padre } \\
\hline & \multicolumn{3}{|c|}{ Adolescentes } & \multicolumn{3}{|c|}{ Progenitores } \\
\hline & Chico & Chica & Total & Chico & Chica & Total \\
\hline Escuela y aprendizajes & 3,11 & 3,40 & 3,27 & $3,43 \uparrow$ & $3,54 \uparrow$ & $3,50 \uparrow$ \\
\hline Televisión & 2,70 & 2,70 & 2,70 & $2,94 \uparrow$ & $2,93 \uparrow$ & $2,94 \uparrow$ \\
\hline Limpieza y orden & 2,52 & 2,72 & 2,63 & $2,51 \downarrow$ & $2,55 \downarrow$ & $2,54 \downarrow$ \\
\hline $\begin{array}{l}\text { Tiempo libre y } \\
\text { actividades de ocio }\end{array}$ & 2,57 & 2,71 & 2,65 & $3,46 \downarrow$ & $3,25 \uparrow$ & $3,33 \uparrow$ \\
\hline Sentimientos & 2,06 & 2,02 & 2,04 & $2,51 \uparrow$ & $2,43 \uparrow$ & $2,45 \uparrow$ \\
\hline Futuro & 2,94 & 3,04 & 2,99 & $3,08 \uparrow$ & $3,02 \downarrow$ & $3,04 \uparrow$ \\
\hline Ordenadores & 2,39 & 2,38 & 2,38 & $2,99 \uparrow$ & $2,83 \uparrow$ & $2,89 \uparrow$ \\
\hline Lo que pasa en el mundo & 2,65 & 2,87 & 2,77 & $3,14 \uparrow$ & $3,12 \uparrow$ & $3,13 \uparrow$ \\
\hline Ropa & 2,38 & 2,33 & 2,35 & $2,42 \uparrow$ & $2,58 \uparrow$ & $2,52 \uparrow$ \\
\hline Comida & 2,66 & 2,82 & 2,75 & $2,80 \uparrow$ & $3,10 \uparrow$ & $2,99 \uparrow$ \\
\hline $\begin{array}{l}\text { La ciudad o pueblo } \\
\text { donde vives }\end{array}$ & 2,58 & 2,55 & 2,56 & $3,11 \uparrow$ & $3,09 \uparrow$ & $3,10 \uparrow$ \\
\hline La vida cotidiana & 2,60 & 2,80 & 2,71 & $3,17 \uparrow$ & $3,17 \uparrow$ & $3,17 \uparrow$ \\
\hline Familia y familiares & 2,89 & 3,23 & 3,08 & $3,37 \uparrow$ & $3,40 \uparrow$ & $3,39 \uparrow$ \\
\hline Otras personas y relaciones & 2,30 & 2,51 & 2,41 & $3,02 \uparrow$ & $3,01 \uparrow$ & $3,02 \uparrow$ \\
\hline $\begin{array}{l}\text { Videoconsolas y } \\
\text { juegos de ordenador }\end{array}$ & 2,01 & 1,58 & 1,78 & $2,58 \uparrow$ & $1,85 \uparrow$ & $2,12 \uparrow$ \\
\hline $\begin{array}{l}\text { Responsabilidad y } \\
\text { otros valores morales }\end{array}$ & 2,69 & 2,95 & 2,83 & $3,11 \uparrow$ & $3,10 \uparrow$ & $3,11 \uparrow$ \\
\hline Religión & 1,62 & 1,61 & 1,61 & $2,24 \uparrow$ & $2,28 \uparrow$ & $2,27 \uparrow$ \\
\hline Deportes & 3,32 & 2,81 & 3,04 & $3,63 \uparrow$ & $3,02 \uparrow$ & $3,25 \uparrow$ \\
\hline Música & 2,53 & 2,63 & 2,59 & $2,96 \uparrow$ & $3,06 \uparrow$ & $3,02 \uparrow$ \\
\hline Revistas & 2,01 & 1,72 & 1,85 & $2,65 \uparrow$ & $2,25 \uparrow$ & $2,40 \uparrow$ \\
\hline
\end{tabular}

$0,014)$, lo que pasa en el mundo $\left(t_{1164}=2,805 ; p=0,005\right)$, la comida $\left(t_{1169}=\right.$ $2,095 ; p=0,036)$, la vida cotidiana $\left(t_{1164}=2,731 ; p=0,006\right)$, la familia y los familiares $\left(t_{1166}=4,649 ; p<0,0005\right)$, otras personas y relaciones $\left(t_{1165}=3,034 ; p\right.$ $=0,002)$ y la responsabilidad y los valores morales $\left(t_{1130}=3,377 ; p=0,001\right)$. Los chicos expresan hablar más frecuentemente que las chicas con su padre de las videoconsolas y los juegos de ordenador $\left(t_{1007}=6,258 ; p<0,0005\right)$, los deportes $\left(t_{1173}=6,280 ; p<0,0005\right)$ y las revistas $\left(t_{1074}=4,141 ; p<0,0005\right)$.

Los progenitores de los chicos consideran significativamente más frecuentes que los progenitores de chicas las conversaciones que el padre mantiene con el chico sobre las videoconsolas y juegos de ordenador $\left(t_{273}=6,352 ; p<0,0005\right)$, sobre los deportes $\left(t_{429}=4,925 ; p<0,0005\right)$ y sobre revistas $\left(t_{429}=3,383 ; p=\right.$ $0,001)$. Los progenitores de chicas, por su parte, consideran las conversaciones del padre significativamente más frecuentes que los progenitores de chicos cuando se refieren a la comida $\left(t_{422}=2,630 ; p=0,009\right)$.

También cuando se trata de hablar con la madre sobre los diferentes aspectos de la vida de los/las adolescentes (Tabla XII), se observan diferencias significativas acerca de los temas tratados según informe un hijo o una hija. Las chicas consideran que la madre habla con ellas más frecuentemente que lo que consideran los chicos sobre la escuela y los aprendizajes $\left(t_{1071}=5,588 ; p<0,0005\right)$, la televisión $\left(t_{1166}=2,772 ; p=0,006\right)$, la limpieza y el orden $\left(t_{1063}=6,957 ; p<0,0005\right)$, el tiempo libre y las actividades de ocio $\left(t_{1165}=4,675 ; p<0,0005\right)$, los sentimientos $\left(t_{1165}=5,714 ; p<0,0005\right)$, el futuro $\left(t_{1165}=3,469 ; p=0,001\right)$, lo que pasa en el 
TABLA XII

Frecuencia en la comunicación con la madre sobre diferentes temas según los adolescentes y según sus progenitores

\begin{tabular}{|c|c|c|c|c|c|c|}
\hline \multirow{3}{*}{ MEDIAS } & \multicolumn{6}{|c|}{ Con la madre } \\
\hline & \multicolumn{3}{|c|}{ Adolescentes } & \multicolumn{3}{|c|}{ Progenitores } \\
\hline & Chico & Chica & Total & Chico & Chica & Total \\
\hline Escuela y aprendizajes & 3,50 & 3,87 & 3,70 & $4,03 \uparrow$ & $4,11 \uparrow$ & $4,08 \uparrow$ \\
\hline Televisión & 2,77 & 2,96 & 2,87 & $3,07 \uparrow$ & $3,30 \uparrow$ & $3,21 \uparrow$ \\
\hline Limpieza y orden & 3,21 & 3,74 & 3,50 & $3,27 \uparrow$ & $3,51 \downarrow$ & $3,42 \downarrow$ \\
\hline $\begin{array}{l}\text { Tiempo libre y } \\
\text { actividades de ocio }\end{array}$ & 2,75 & 3,11 & 2,95 & $3,73 \uparrow$ & $3,80 \uparrow$ & $3,77 \uparrow$ \\
\hline Sentimientos & 2,36 & 2,84 & 2,62 & $3,12 \uparrow$ & $3,39 \uparrow$ & $3,29 \uparrow$ \\
\hline Futuro & 3,09 & 3,35 & 3,23 & $3,37 \uparrow$ & $3,43 \uparrow$ & $3,41 \uparrow$ \\
\hline Ordenadores & 2,20 & 2,20 & 2,20 & $2,81 \uparrow$ & $2,90 \uparrow$ & $2,87 \uparrow$ \\
\hline Lo que pasa en el mundo & 2,73 & 2,98 & 2,86 & $3,29 \uparrow$ & $3,30 \uparrow$ & $3,29 \uparrow$ \\
\hline Ropa & 3,02 & 3,75 & 3,42 & $3,40 \uparrow$ & $4,24 \uparrow$ & $3,92 \uparrow$ \\
\hline Comida & 3,03 & 3,34 & 3,20 & $3,53 \uparrow$ & $3,92 \uparrow$ & $3,77 \uparrow$ \\
\hline $\begin{array}{l}\text { La ciudad o pueblo } \\
\text { donde vives }\end{array}$ & 2,63 & 2,66 & 2,65 & $3,26 \uparrow$ & $3,27 \uparrow$ & $3,27 \uparrow$ \\
\hline La vida cotidiana & 2,73 & 3,00 & 2,88 & $3,41 \uparrow$ & $3,53 \uparrow$ & $3,48 \uparrow$ \\
\hline Familia y familiares & 3,09 & 3,48 & 3,31 & $3,74 \uparrow$ & $3,78 \uparrow$ & $3,76 \uparrow$ \\
\hline Otras personas y relaciones & 2,51 & 2,97 & 2,76 & $3,23 \uparrow$ & $3,52 \uparrow$ & $3,41 \uparrow$ \\
\hline $\begin{array}{l}\text { Videoconsolas y juegos } \\
\text { de ordenador }\end{array}$ & 1,92 & 1,52 & 1,70 & $2,53 \uparrow$ & $1,99 \uparrow$ & $2,20 \uparrow$ \\
\hline $\begin{array}{l}\text { Responsabilidad y } \\
\text { otros valores morales }\end{array}$ & 2,86 & 3,28 & 3,09 & $3,40 \uparrow$ & $3,46 \uparrow$ & $3,43 \uparrow$ \\
\hline Religión & 1,76 & 1,79 & 1,78 & $2,43 \uparrow$ & $2,56 \uparrow$ & $2,51 \uparrow$ \\
\hline Deportes & 2,72 & 2,58 & 2,65 & $3,32 \uparrow$ & $3,00 \uparrow$ & $3,12 \uparrow$ \\
\hline Música & 2,64 & 2,99 & 2,83 & $3,27 \uparrow$ & $3,42 \uparrow$ & $3,36 \uparrow$ \\
\hline Revistas & 2,03 & 2,21 & 2,13 & $2,90 \uparrow$ & $2,87 \uparrow$ & $2,88 \uparrow$ \\
\hline
\end{tabular}

mundo $\left(t_{1111}=3,366 ; p=0,001\right)$, la ropa $\left(t_{1053}=9,674 ; p<0,0005\right)$, la comida $\left(t_{1165}=4,129 ; p<0,0005\right)$, la vida cotidiana $\left(t_{1161}=3,615 ; p<0,0005\right)$, la familia y los familiares $\left(t_{1164}=5,657 ; p<0,0005\right)$, las otras personas y relaciones $\left(t_{1096}=\right.$ $6,131 ; p<0,0005)$, la responsabilidad y los valores morales $\left(t_{1161}=5,319 ; p<\right.$ $0,0005)$, la música $\left(t_{1150}=4,426 ; p<0,0005\right)$ y las revistas $\left(t_{1161}=2,370 ; p=\right.$ 0,018). Los chicos consideran que su madre habla con ellos más frecuentemente que lo que consideran las chicas sobre las videoconsolas y los juegos de ordenador $\left(t_{983}=6,119 ; p<0,0005\right)$.

Cuando se plantea esta misma cuestión a sus progenitores, los de las chicas manifiestan que la madre habla con su hija con una frecuencia significativamente mayor de lo que los progenitores de los chicos manifiestan que habla con su hijo sobre la televisión $\left(t_{452}=2,329 ; p=0,020\right)$, los sentimientos $\left(t_{454}=2,395\right.$; $p$ $=0,017)$, la ropa $\left(t_{272}=8,784 ; p<0,0005\right)$, la comida $\left(t_{309}=3,828 ; p<0,0005\right)$ y otras personas y relaciones $\left(t_{450}=2,810 ; p=0,005\right)$. Los progenitores de los chicos tienden a informar de una frecuencia mayor de comunicación de la madre con su hijo con respecto a las videoconsolas y juegos de ordenador $\left(t_{342}=4,770 ; p\right.$ $<0,0005)$ y los deportes $\left(t_{451}=2,707 ; p=0,007\right)$ que los progenitores de chicas.

\section{Satisfacción con las conversaciones}

Las tablas que vienen a continuación (Tablas XIII, XIV, XV y XVI) muestran las medias de las puntuaciones dadas a la satisfacción proporcionada por las conversaciones con distintos interlocutores acerca de las actividades realizadas con cada 
medio según el o la adolescente, y, al mismo tiempo, la que los propios progenitores atribuyen a su hijo o hija. En todos los casos las respuestas se han obtenido mediante escalas tipo Likert del 1 (No me gusta nada) al 5 (Me gusta muchísimo).

TABLA XIII

Satisfacción de los/las adolescentes al hablar con el padre sobre medios audiovisuales y atribución que los progenitores bacen a sus bijos/as

\begin{tabular}{llcccc}
\hline MEDIAS & & Televisión & Ordenador & Internet & Videojuegos \\
\hline Satisfacción de los & Chico & 3,24 & 2,79 & 2,66 & 2,56 \\
chicos/as al hablar & Chica & 3,16 & 2,80 & 2,65 & 1,95 \\
con el padre & Total & 3,20 & 2,80 & 2,65 & 2,26 \\
\hline $\begin{array}{l}\text { Atribución de los } \\
\text { progenitores de la }\end{array}$ & Chico & $3,22 \downarrow$ & $3,20 \uparrow$ & $3,06 \uparrow$ & $2,71 \uparrow$ \\
$\begin{array}{l}\text { satisfacción del chico/a } \\
\text { al hablar con el padre }\end{array}$ & Total & $3,16 \rightarrow$ & $3,09 \uparrow$ & $2,88 \uparrow$ & $1,99 \uparrow$ \\
\hline
\end{tabular}

En la satisfacción con las conversaciones con el padre (Tabla XIII), la única diferencia estadísticamente significativa que hemos observado entre chicos y chicas, a favor de los primeros, se refiere a la que proporciona hablar con él sobre videojuegos $\left(t_{693}=5,998 ; p<0,0005\right)$.

En lo que respecta a las respuestas de los progenitores, observamos que éstos tienden a sobrevalorar la satisfacción de los chicos/as al hablar con el padre acerca del ordenador y de Internet $\left(t_{270}=4,902 ; p<0,0005\right.$ y $t_{238}=2,858 ; p=0,005$, respectivamente).

Si tenemos en cuenta si los progenitores que han contestado el cuestionario lo son de un chico o una chica, observamos como los primeros atribuyen significativamente mayor satisfacción a su hijo al hablar con el padre sobre los videojuegos $\left(t_{247}=4,847 ; p<0,0005\right)$ que los progenitores de chicas hacen respecto a su hija.

TABLA XIV

Satisfacción de los/las adolescentes al bablar con la madre sobre medios audiovisuales y atribución que los progenitores bacen a sus hijos/as

\begin{tabular}{llcccc}
\hline MEDIAS & & Televisión & Ordenador & Internet & Videojuegos \\
\hline Satisfacción de los & Chico & 3,28 & 2,61 & 2,55 & 2,29 \\
chicos/as al hablar & Chica & 3,53 & 2,78 & 2,76 & 1,87 \\
con la madre & Total & 3,42 & 2,71 & 2,67 & 2,08 \\
\hline $\begin{array}{l}\text { Atribución de los } \\
\text { progenitores de la }\end{array}$ & Chico & $3,30 \uparrow$ & $3,21 \uparrow$ & $3,04 \uparrow$ & $2,65 \uparrow$ \\
$\begin{array}{l}\text { satisfacción del chico/a } \\
\text { al hablar con la madre }\end{array}$ & Total & $3,50 \downarrow$ & $3,20 \uparrow$ & $3,08 \uparrow$ & $2,08 \uparrow$ \\
\hline
\end{tabular}

Cuando esta comunicación es con la madre (Tabla XIV), vemos que las diferencias significativas en la satisfacción entre chicos y chicas se dan respecto a la televisión, Internet y los videojuegos, a favor de las chicas para los dos primeros medios (televisión: $t_{969}=3,483 ; p=0,001$ e Internet: $t_{816}=2,252 ; p=0,025$ ) y a favor de los chicos para el tercer medio $\left(t_{715}=4,511 ; p<0,0005\right)$.

En lo que se refiere a la atribución de satisfacción de los progenitores, se han encontrado diferencias significativas para el ordenador $\left(t_{287}=5,487 ; p<0,0005\right)$, 
Internet $\left(t_{261}=2,484 ; p=0,014\right)$ y los videojuegos $\left(t_{201}=2,104 ; p=0,037\right)$, sobrevalorando los progenitores las satisfacciones de sus hijos/as en las conversaciones con la madre sobre los tres medios.

Los progenitores de chicas atribuyen a su hija mayor satisfacción en las conversaciones con la madre sobre televisión $\left(t_{340}=2,092 ; p=0,037\right)$ y menor satisfacción cuando son sobre videojuegos $\left(t_{318}=3,872\right.$; $\left.p<0,0005\right)$ que los progenitores de chicos a su hijo.

TABLA XV

Satisfacción de los/las adolescentes al hablar con los amigos/as sobre medios audiovisuales y atribución que los progenitores bacen a sus hijos/as

\begin{tabular}{|c|c|c|c|c|c|}
\hline MEDIAS & & Televisión & Ordenador & Internet & Videojuegos \\
\hline \multirow{3}{*}{$\begin{array}{l}\text { Satisfacción de los } \\
\text { chicos/as al hablar } \\
\text { con los amigos/as }\end{array}$} & Chico & 3,96 & 4,01 & 4,00 & 4,10 \\
\hline & Chica & 4,07 & 3,71 & 3,99 & 2,60 \\
\hline & Total & 4,02 & 3,84 & 3,99 & 3,37 \\
\hline \multirow{2}{*}{$\begin{array}{l}\text { Atribución de los } \\
\text { progenitores de la } \\
\text { satisfacción del chico/a } \\
\text { al hablar con los amigos/as }\end{array}$} & $\begin{array}{l}\text { Chico } \\
\text { Chica }\end{array}$ & $\begin{array}{l}3,55 \downarrow \\
3,82 \downarrow\end{array}$ & $\begin{array}{l}3,98 \downarrow \\
3,81 \uparrow\end{array}$ & $\begin{array}{l}3,92 \downarrow \\
4,04 \uparrow\end{array}$ & $\begin{array}{l}3,98 \downarrow \\
2,87 \uparrow\end{array}$ \\
\hline & Total & $3,73 \downarrow$ & $3,87 \uparrow$ & $4,00 \uparrow$ & $3,31 \downarrow$ \\
\hline
\end{tabular}

Con respecto a la satisfacción que chicos y chicas experimentan cuando hablan con los amigos/amigas (Tabla XV) observamos que los chicos se muestran significativamente más satisfechos que las chicas al hablar del ordenador $\left(t_{1014}=\right.$ $4,112 ; p<0,0005)$ y de los videojuegos $\left(t_{820}=16,617 ; p<0,0005\right)$. En el caso de las atribuciones hechas por sus progenitores a esta satisfacción, se observan diferencias estadísticamente significativas cuando se refiere a conversaciones mantenidas con los amigos/as sobre la televisión $\left(t_{318}=5,195 ; p<0,0005\right)$, considerando los progenitores que la satisfacción de sus hijos/as es significativamente menor de lo que los propios adolescentes manifiestan.

Los progenitores de chicas atribuyen a su hija mayor satisfacción en las conversaciones con sus amigos y/o amigas sobre la televisión $\left(t_{347}=2,372 ; p=\right.$ $0,018)$ y menor satisfacción cuando son sobre videojuegos $\left(t_{326}=7,750 ; p<\right.$ $0,0005)$ en comparación con lo que los progenitores de chicos atribuyen a su hijo.

Finalmente, la inmensa mayoría tanto de chicos como de chicas informan de satisfacciones ínfimas cuando hablan de cualquier actividad con los medios audiovisuales con algún profesor o profesora (Tabla XVI), difiriendo de forma significativa unos y otras en los siguientes medios: ordenador $\left(t_{634}=3,723 ; p<\right.$

TABLA XVI

Satisfacción de los/las adolescentes al hablar con algún/a profesor/a sobre medios audiovisuales y atribución que los progenitores hacen a sus hijos/as

\begin{tabular}{lccccc}
\hline MEDIAS & & Televisión & Ordenador & Internet & Videojuegos \\
\hline $\begin{array}{llccc}\text { Satisfacción de los } \\
\text { chicos/as al hablar }\end{array}$ & Chico & 1,81 & 1,78 & 1,67 & 1,39 \\
con el/la profesor/a & Total & 1,91 & 2,11 & 2,08 & 1,24 \\
\hline $\begin{array}{l}\text { Atribución de los } \\
\text { progenitores de la }\end{array}$ & Chico & $2,14 \uparrow$ & $2,90 \uparrow$ & $2,81 \uparrow$ & $1,90 \uparrow$ \\
$\begin{array}{l}\text { satisfacción del chico/a } \\
\text { al hablar con el/la profesor/a }\end{array}$ & Total & $2,30 \uparrow$ & $2,81 \uparrow$ & $2,78 \uparrow$ & $1,71 \uparrow$ \\
\hline
\end{tabular}


$0,0005)$ e Internet $\left(t_{609}=4,549 ; p<0,0005\right)$ estando más satisfechas las chicas, $\mathrm{y}$ los videojuegos $\left(t_{466}=2,147 ; p=0,032\right)$ estando más satisfechos los chicos.

Los progenitores atribuyen satisfacciones significativamente muy superiores a su hijo o hija que las informadas por éste o ésta, en relación con tales conversaciones cuando éstas se refieren a todos los medios estudiados: televisión $\left(t_{117}=\right.$ $2,442 ; p=0,016)$, ordenador $\left(t_{127}=6,641 ; p<0,0005\right)$, Internet $\left(t_{123}=6,708 ; p\right.$ $<0,0005)$ y los videojuegos $\left(t_{85}=3,067 ; p=0,003\right)$. No existen diferencias significativas entre las respuestas de los progenitores de chicos y los de chicas.

\section{Discusión}

Nuestro análisis se ha centrado en explorar, por una parte, las diferencias de género en las respuestas de chicos y chicas y por otra las diferencias entre generaciones (progenitores e hijos/as) dentro de la misma familia.

El interés actual y el nivel de información de los y las adolescentes respecto a los medios audiovisuales explorados son, en general, claramente distintos según el género. Algunos estudios transculturales (Krotz y Hasebrink, 2001) han sugerido que los chicos parecen estar más orientados hacia la tecnología que las chicas. Así mismo, Oksman y Rautiainen (2002) hablan de determinismo tecnológico y miedo a la tecnología, atribuido más a las chicas que a los chicos, al referirse a los ordenadores.

Nuestros resultados muestran en este sentido como los chicos manifiestan estar más informados e interesados por el ordenador, las videoconsolas y los juegos de ordenador. Sin embargo, y confirmando tendencias ya detectadas los últimos años en algunos países (Suess et al., 1998), las chicas de nuestra muestra manifiestan estar más informadas e interesadas que los chicos acerca de algunas nuevas tecnologías, especialmente el teléfono móvil. Así, parece constatarse una vez más que la cultura de los chicos está más relacionada con los juegos y la acción en general y la de las chicas está más basada en las relaciones y las conversaciones, hecho reafirmado por su mayor uso de chats y correo electrónico, de entre las facilidades que ofrece un ordenador.

Bovill y Livingstone (2001) sugieren que son las diferencias en los estilos de socialización de chicos y chicas los que afectan el uso que unos y otras hacen de los medios audiovisuales. En uno de sus estudios pudieron observar que las chicas hablaban más horas por teléfono y, además, lo hacían por motivos diferentes. Las conversaciones de los chicos estaban más orientadas a resolver objetivos específicos, mientras que las de las chicas estaban más orientadas a mantener el contacto social.

Tecnologías como el ordenador, y algunas de sus facilidades más recientes, como Internet, son utilizadas de manera habitual, tanto por los chicos/as, como por buena parte de los progenitores de esta muestra. Sin embargo, los resultados muestran que chicos y chicas utilizan el ordenador e Internet para diferentes usos y funciones. Parecer ser que a los chicos les atraen más aquellas funciones relacionadas con los juegos, la música y las películas, mientras que a las chicas aquellas que les son útiles como medio para organizar las actividades diarias, así como, para constituir y mantener sus redes sociales (prefieren escribir, chatear o enviar correos electrónicos).

Por su parte, los progenitores en general muestran un perfil distinto y notoriamente más restringido de usos de estos medios, que su propio hijo o hija.

Hemos explorado con escalas bipolares cómo evalúan los/las adolescentes sus experiencias con los diferentes medios. Los chicos varones de la muestra han manifestado sensaciones más intensas (ya sean de aprendizaje, de aprovechamiento del tiempo, de utilidad, de actividad y/o de acompañamiento) que las 
chicas con la televisión, el ordenador, Internet y los videojuegos. Del Río (1999), basándose en un estudio de Ortega (1992), plantea que las actividades que hacen niños y niñas desde pequeños parecen muy diferenciadas. Los niños parecen practicar mucho más menudo juegos de desgaste físico o manipulativos de intenso feed-back perceptivo-motor (como los electrónicos), mientras que las niñas prefieren juegos más sedentarios.

Cuando los progenitores realizan atribuciones acerca del interés e información que creen que su hijo o hija tiene sobre los diferentes medios, observamos una tendencia general a sobrevalorarlos.

Comparando un abanico de experiencias evaluadas por el chico o chica, con las atribuciones de sus propios progenitores acerca de dichas experiencias, respecto al uso de diferentes medios audiovisuales, observamos que, en general, estos últimos tienden a sobrevalorar las experiencias positivas (concretamente, la diversión y el aprendizaje) del primero/a respecto al uso del ordenador, mientras que tienden a subvalorar las relacionadas con mirar la televisión.

Cuando preguntamos a los y las adolescentes sobre qué actividades de la vida diaria bablan con más frecuencia con cada uno de sus progenitores, observamos que en las respuestas se aprecian diferencias según el sexo del hijo/a, tanto si los informantes son los progenitores, como si es el propio chico o chica. En algunos casos las diferencias que aparecen son congruentes entre si, pero en otros no, evidenciando la existencia de sesgos perceptivos.

Garitaonandia, Juaristi y Olega (2001) ya señalaban diferencias en los temas de conversación según el género de los/as adolescentes: los temas de conversación de interés para los chicos son más uniformes y están más orientados a la acción; mientras que, los de las chicas son más variados y más orientados hacia las relaciones sociales. Nuestros resultados apoyan esta idea y la de que, además, hay sesgos perceptivos de los observadores según el género del observado/a, como también han señalado Moreno y Sastre (2000).

En general, tanto para las conversaciones con el padre como con la madre, los progenitores tienden a sobredimensionar la frecuencia de todos los temas de conversación, si comparamos sus respuestas con las del propio hijo/a, exceptuando en los temas de limpieza y orden.

La satisfacción con las conversaciones sobre las actividades de interés realizadas con los medios audiovisuales resulta quizás el tema más relevante de los que hemos explorado. Cabe destacar la gran diferencia de satisfacción con las conversaciones que los y las adolescentes manifiestan cuando hablan con adultos y cuando lo hacen con iguales en general, observándose la más alta insatisfacción con los profesores. Por contraste, hablar de los medios audiovisuales con los amigos/as se nos muestra como una actividad muy importante. Observando las tablas XIII, XIV y XVI lo que más llama la atención es, sin duda, la tendencia general de los progenitores a sobredimensionar la satisfacción de su hijo o hija con la mayoría de las conversaciones con los adultos sobre sus actividades con NTICs.

Las relaciones con los amigos/as son una manera que tienen los chicos/as para desarrollar un sentido de sí mismo respecto de la sociedad. Según James (1993) la participación de los chicos y chicas en una red social de relaciones entre iguales les ayuda a construir la identidad y el sentido de sí mismo. Hartup (1992), que ha investigado exhaustivamente las relaciones de amistad en la infancia, observa cómo hacer amigos/as y mantener estas relaciones es considerado por los propios chicos y chicas como uno de los logros más importantes de la infancia y la adolescencia.

Los y las adolescentes pasan buena parte de su tiempo libre en casa con los medios audiovisuales, sobre todo en su propio espacio privado (frecuentemente 
en sus habitaciones) más que en el común o familiar. De esta forma, los chicos y chicas pasan un importante tiempo de sus vida aislados, dificultando que los progenitores puedan conversar con ellos/as o regular el uso que hagan de los medios. En este sentido, Bovill y Livingstone (2001) apuntan que esto puede tener dos consecuencias: por una lado, el aislamiento, que puede llegar a preocupar a los progenitores; y por otro, el aumento de la privacidad, experimentada por los y las adolescentes como menor control de los adultos.

Respecto a la baja satisfacción con mantener conversaciones con sus profesores es una constante que se repite en anteriores estudios que hemos realizado (Casas et al., 2000; 2002). Algunos estudios sugieren que esto es debido a la poca integración que hay en las escuelas de las nuevas tecnologías. Por ejemplo, en el caso de España, Italia y Alemania la nuevas tecnologías son introducidas hacia al final de la escolarización en comparación con otros países como Inglaterra, Dinamarca y Holanda donde éstas son introducidas desde la enseñanza primaria (Suess et al., 1998; Süss, 2001). Aunque también se ha señalado como explicación el alejamiento de la escuela de la vida social cotidiana que experimentan los chicos y chicas (Casas et al., 2000).

Globalmente podemos resumir nuestros resultados como una confirmación de que existen interacciones entre el género y el uso de NTICs, en muchos aspectos reproduciendo los estereotipos de género mayoritarios en nuestra cultura occidental. Sin embargo, estas diferentes "culturas de género" parecen mediatizadas por unas "culturas generacionales". No sólo se observa que los adolescentes entre 12 y 16 años utilizan más las NTICs que los adultos, sino que el contexto social sobre el que tiene impacto dicho uso es fundamentalmente el de la red de iguales. Es con los iguales con quienes chicos y chicas obtienen mayor satisfacción relatando las experiencias de tal uso.

Las atribuciones de los progenitores acerca de usos, experiencias y preferencias de su propio hijo o hija con las NTICs son a menudo significativamente distintos de los informados por el chico o chica. Este hecho, sumado a la insatisfacción con las conversaciones con cualquier adulto sobre estos temas y a los sesgos perceptivos de los adultos sobre las experiencias de su hijo o hija con los medios, parece sugerir la necesidad de un mayor acercamiento intergeneracional, que incluya una mayor presencia de los temas de alta motivación para los chicos y chicas adolescentes, como es el caso de las nuevas tecnologías audiovisuales, en las conversaciones entre los adultos y los más jóvenes. Ello requiere que los adultos aceptemos el intercambio en campos en donde nuestras habilidades y experiencias no superan necesariamente las de los más jóvenes, como tradicionalmente estamos acostumbrados a que suceda.

\section{Referencias}

Bovill, M. \& Livingstone, S. (2001). Bedroom Culture and Privatization of Media Use. En S. Livingstone \& M. Bovill (Eds.), Children and Their Changing Media Environment. A European Comparative Study (pp. 179-200). Londres: Lawrence Erlbaum.

Bressand, A. \& Distler, C. (1995). La planète relationnelle. París: Flammarion. [Trad. cast.: El planeta relacional. Barcelona: Proa, 1997].

Buckingham, D. (2000). After the death of childhood. Growing up in the age of electronic media. Policy Press in association with Blackwell. [Trad. cast: Crecer en la era de los medios electrónicos. Madrid: Morata, 2002].

Buckingham, D. (2003). Multimedia childhoods. En M. J. Kehily \& J. Swann (Eds.), Children's cultural worlds (pp. $184-$ 222). Chichester: Wiley/Open University.

CASAS, F. (1995). Infancia y medios de comunicación. Cuadernos de Pedagogía, 239, 17-19.

CASAS, F. (1998). Children and the audiovisual culture. World Forum on Children's television. Barcelona, Noviembre.

CASAS, F. (1999). Children, media and the relational planet: some reflections from the European context. Child Studies, 1 , March, 119-146. International Center for Child Studies. Konan Women's University. Kobe, Japón (Formato electrónico: http://www.childresearch.net/Key/Kmedia/PCE/index.html).

Cassas, F., Alsinet, C., Pérez Tornero, J. M., Figuer, C., Leiva, E., González, M. \& Pascual, S. (2000). Las tecnologías de la información y la comunicación entre los padres y los hijos. Intervención Psicosocial, 9 (3), 335-360. 
Casas, F., Figuer, C., GonzÁlez, M. \& Alsinet, C. (2002). ¿Qué coincidencias y discrepancias tienen los jóvenes y sus padres ante los medios? Comunicar, 18, 47-52.

DEL Río, P. (1999). El cambio histórico-cultural y las identidades de género: los/las adolescentes españoles ante la diferencia de sexo. Cultura y Educación, 14/15, 147-176.

DEL Río, P., AlvareZ, A. \& DEL Río, M. (2004). Pigmalión. Informe sobre el impacto de la televisión en la infancia. Madrid: Fundación Infancia y Apredizaje.

Garitaonandia, C., Juaristi, P. \& Olega, J. A. (2001). Media Genres and Content Preference. En S. Livingstone \& M. Bovill (Eds.), Children and Their Changing Media Environment. A European Comparative Study (pp. 141-158). Londres: Lawrence Erlbaum .

HARtuP, W. (1992). Friendships and their development significance. En H. McGurk (Ed.), Childhood social development: contemporany perspectives (pp. 175-205). Hove: Lawrence Erlbaum.

JAMES, A. (1993). Childhood identities: self and social relationships in the experience of the child. Edimburgo: Edinburg University Press.

Krotz, F. \& Hasebrink, U. (2001). Who are the New Media User? En S. Livingstone \& M. Bovill (Eds.), Children and Their Changing Media Environment. A European Comparative Study (pp. 245-262). Londres: Lawrence Erlbaum.

LLULL, J. (1980). The social uses of television. Human Communication Research, 6 (3), 197-209.

McLuHAN, M. (1977). La comprensión de los medios como extensiones del hombre. México: Diana.

MorenO \& SASTRE, G. (2000). Repensar la ética desde una perspectiva de género. Intervención Psicosocial, 9 (1), 35-48.

OHMAE, K. (1995). The end of the nation state. Nueva York: Harper Collins.

Oksman, V. \& Rautiainen, P. (2002). Toda mi vida en la palma de mi mano: La comunicación móvil en la vida diaria de niños y adolescentes de Finlandia. Revista de Estudios de Juventud, Monográfico Juventud y teléfonos móviles, 57, 25 -32.

ORTEGA, R. (1992). El juego infantil y la construcción social de conocimiento. Sevilla: Alfar.

PAPERT, S. (1996). The connected family: Bridging the digital generation gap. Atlanta, GA: Longstreet.

Postman, N. (1983). The Disappearance of Childhood. Londres: W. H. Wallen.

Postman, N. ( 1986). Amusing Ourselves to Death. Londres: Methuen.

Suess, D., Suoninen, A., Garitaonandia, C., Juaristi, P., Koikkalainen, R. \& Oleaga, J. A. (1998). Media use and the relationship of children and teenagers with their peer groups. European Journal of Communication, 13 (4), 521-538.

Süss, D. (2001). Computers and the Internet in School: Closing Knowledge Gap? En S. Livingstone \& M. Bovill (Eds.), Children and Their Changing Media Environment. A European Comparative Study (pp. 221-242). Londres: Lawrence Erlbaum.

Torres, E., CONDE, E. \& Ruiz, C. (2002). Desarrollo bumano en la sociedad audiovisual. Madrid: Alianza. 